\title{
Review of: "China's deserts greening and response to climate variability and human activities"
}

\author{
Shaobo Sun ${ }^{1}$ \\ 1 Tianjin University
}

Potential competing interests: The author(s) declared that no potential competing interests exist.

The authors examined variations and drivers of NDVI in the deserts of China. The paper is helpful for understanding the impacts of climate changes and human activities on vegetation dynamics in arid regions. However, it was not good in writing, and was not well concluded. The language problems must be addressed. For example, use of definite article "the". The conclusion also should be improved.

Special comments are as follows,

\section{Abstract}

1. correlation coefficients - correlation coefficient

2. the NDVI - NDVI

3. all four seasons - all seasons

4. "The NDVI were higher in sum- mer and autumn than in spring and winter. " This sentence was meaningless $\square$ delete it.

5. Both the monthly - Both monthly

6. on the southern edge - in the southern edge

7. at the monthly and seasonal scales - at monthly and seasonal scales

8. at the monthly - at monthly

Introduction

9. To clarify - clarifying

10. "can identify low biomass and an increase in vegetation cover than other indexes" - This is difficult for understanding. I suggest "can identify vegetation with low biomass"

11. Seasonal differences were seen - Seasonal differences were found

12. "Among various environmental factors, variations in temperature and precipitation, which are forms of climate variability, are considered" - "Among various environmental factors, temperature and precipitation are considered"

13. "The NDVI is an effective indicator of conditions affecting vegetation growth and can reflect the impacts of environmental and anthropogenic factors. " please delete this sentence, it was meaningless. 


\section{Data sources}

14. "In this study, the NDVI from MOD13A2 was used as an indicator of vegetation activity. The NDVI dataset was sourced from the MODIS " repetitive sentences, I suggest "The NDVI data used was the Moderate Resolution Imaging Spectroradiometer $\square$ MODIS $\square$ NDVI product (MOD13 A2 $\square$ "

15. Caption of Fig. 1: deserts from 2000 to 2017 - deserts during 2000 to 2017

Methods

16. Correlation analysis of the NDVI and climatic factors. - Correlation analysis between NDVI and climatic factors.

17. in previous studies to evaluate the relationships - in previous studies to examine the relationships

18. on the NDVI of deserts - on the NDVI in deserts

Results

19. "summer (0.1005) >autumn (0.0837) >spring (0.0729)> winter (0.0601). ", Why the NDVI was an effective value in winter? I am not sure if this is reasonable. In the desert regions, NDVI should very small during the non-growing seasons.

20. Fig. 2: I think Fig.2d-f should be moved to section that focuses on analyzing the impacts of climate on NDVI.

21. Fig. 3: "trends (red lines) " $\square$ it was from Theil-Sen estimator? Please clarify this.

22. "in deserts and to compare the impact of different time scales on the correlations, as presented in Table 3." - in deserts (Table 3).

23. temperature and precipitation on the NDVI - temperature and precipitation on NDVI

24. Lagged effect of climatic factors on NDVI - please clarify how to analyze the lagged effect in the method section.

25. Fig. 6: Spatial distribution of the Pearson correlation coefficient for the relationships between the NDVI and climatic factors. - Spatial distribution of the Pearson correlation coefficient between NDVI and climatic factors.

\section{Discussion}

26. "The lagged effects of climatic factors on NDVI were not observed at the monthly scale, which may be because the time scale was not fine enough that the lagged effect might be less than one month. ", In addition to the time scale problem, the fast response of vegetation to climate (especially precipitation) might be also an important reason.

\section{Conclusions}

27. “This study analyzed greening and browning trends in China's deserts from 2000 to 2017 shown in the monthly, seasonal, and annual NDVI both temporally and spatially and mea- sured the effects of climate variability and human activities by correlation analysis and residual analysis. ", The sentence is too long 
and too difficult to understand. Please simplify it.

28. "At the annual scale, climatic factors had limited explanatory power for NDVI variations, and only precipitation had a significant effect on NDVI, and the effect of precipitation was stronger than that of temperature. "I think one of the most important finding in the study is that the increasing precipitation caused greening in the deserts of China. The also should firstly highlight this point in both conclusion and abstract section.

29. In addition to above comments, the authors's results showed that temperature was closely correlated with NDVI at monthly scale, while it was not significant for both spatial averaged data and at spatial. Why there was this difference? Please discuss/explain this in the discussion section. 